\title{
Distribution of Candida Species and Molecular Typing of $C$. albicans Isolates in a Mexico City Tertiary Care Hospital from 2011 to 2013
}

\author{
Haydee Torres Guerrero ${ }^{*}$, Israel Martínez Espinosa ${ }^{1}$, Misael Gonzales Ibarra ${ }^{2}$, \\ Magdalena Aguirre García ${ }^{1}$ \\ ${ }^{1}$ Unidad de Medicina Experimental, Facultad de Medicina, Universidad Nacional Autónoma de México, \\ México D.F., México \\ ${ }^{2}$ Laboratorio de Inmunoalergología y Micología Médica, División de Investigación, Hospital Juárez de México, \\ México D.F., México \\ Email: "haydeet@unam.mx, "htorresguerrero@yahoo.com
}

Received 4 March 2016; accepted 19 June 2016; published 22 June 2016

Copyright (C) 2016 by authors and Scientific Research Publishing Inc.

This work is licensed under the Creative Commons Attribution International License (CC BY). http://creativecommons.org/licenses/by/4.0/

(c) (†) Open Access

\begin{abstract}
The incidence of yeast infections has increased significantly over the past few years, constituting a leading cause of morbidity and mortality among hospitalised patients. The rapid identification of candidiasis is important for the clinical management of patients and to facilitate tracing the sources of infections in hospitalized patients. Here, we report a retrospective, single-centre study of Candida spp. distribution and antifungal susceptibility from January 2011 to May 2013 at a hospital in México City, regarding the importance of elucidating the identity of the infection-causing Candida species in order to improve prophylactic measures and treatment. Clinical data were collected from patient medical records and the laboratory database. Isolates were initially identified using standard mycology techniques, and then confirmed by PCR-based system using amplification of intergenic spacers (rDNA ITS) and restriction length polymorphism of PCR products after sequence-specific enzymatic cleavage (PCR-RFLP). We observed no shift from $C$. albicans to nonalbicans Candida species: Candida albicans $(73.7 \%)$ was the most prevalent species isolated, while C. dubliniensis was not identified in this study. Antifungal susceptibility was determined using FUNGITEST ${ }^{\circledR} ; 17.4 \%$ of $C$. albicans isolates were resistant to fluconazole and $21.7 \%$ to itraconazole. Multiplex PCR microsatellite analysis of the clinical $C$. albicans isolates using primers for the CAI, CAIII and CAVI loci identified 29 different alleles for CAI, 8 alleles for CAIII and 31 for CAVI. The combined discriminatory power of these three microsatellites was 0.98 , which was considered reliable for molecular typing. Genetic analysis of these isolates revealed a clonal population with a total of 62 genotypes from the examined isolates.
\end{abstract}

${ }^{*}$ Corresponding author.

How to cite this paper: Torres Guerrero, H., Martínez Espinosa, I., Gonzales Ibarra, M. and Aguirre García, M. (2016) Distribution of Candida Species and Molecular Typing of C. albicans Isolates in a Mexico City Tertiary Care Hospital from 2011 to 2013. Open Journal of Medical Microbiology, 6, 66-79. http://dx.doi.org/10.4236/ojmm.2016.62010 


\section{Keywords}

\section{Candidiasis, rDNA-ITS, RFLP, Microsatellites, Molecular Typing, CAI, Candida spp.}

\section{Introduction}

The incidence of yeast infections in hospitals has greatly increased in recent decades; these infections are a major cause of morbidity and mortality in patients. Candidiasis is common among critically ill patients hospitalised in the intensive care unit (ICU) for over a week, especially in those with immune deficiency and severe underlying diseases. In addition, the incidence of candidiasis has increased with the use of broad-spectrum antibiotics treatment, chemotherapy and invasive medical devices [1]. Since several Candida spp. are present in the humanassociated microbes colonisation develops in up to $80 \%$ of critically ill patients; however, invasive candidiasis is documented in only $5 \%-10 \%$ of them [2].

Common diagnostic criteria of candidiasis include the observation of filamentous forms or clusters of budding yeasts in biological samples or during post mortem histopathological examination, a serum titre of $\beta$-1, 3-glucan and isolation of fungi from sterile samples [3]. In vitro studies have shown that the pseudo hyphae and hyphae of Candida albicans and C. tropicalis enable invasion of human and murine cells; these forms are considered as indicative of infection [4] [5] in most Candida species except for C. glabrata.

Although C. albicans is the most common candidiasis-causing species, approximately $45 \%$ of cases are caused by non-albicans species that have emerged as colonisers and pathogens [6]. The appearance of these non-albicans species is of special concern because of their intrinsic resistance to antifungal drugs. These features represent a clinical problem since inappropriate empirical antifungal therapy may result in adverse outcomes. Rapid diagnosis and species identification are required for early and accurate treatment that will eventually reduce the mortality rate of patients. Phenotypic identification is routinely used in clinical laboratories, but these methods can take 48 to $72 \mathrm{~h}$ to identify the pathogen to the species level, and the evaluation of these characteristics requires expertise [7].

Many attempts have been made over the last decade to develop techniques that can accurately identify species and distinguish between endemic and sporadic strains [8]-[10], such techniques would enable detection of the origin of infection and identification of strains involved in outbreaks. Transmission of Candida spp. occurs via direct contact; however, there have been worldwide reports of cross infection due to health care workers within hospitals.

Molecular methods are more reliable and have been used extensively for diagnosis and identification with high levels of sensitivity, specificity and reproducibility; this is advantageous for determining and optimising treatment early in the infection, and is important for elucidating the distribution of Candida species. For example, microsatellite analysis based on the amplification of highly polymorphic short tandem repeats can be used to generate characteristic profiles for different alleles of a specific locus; the high differentiation power (DP) of this technique permits studies of nosocomial transmission using the CAI, CAIII and CAVI loci [10].

In México, candidiasis surveillance programs have been sporadic, and data regarding the distribution of different Candida species in tertiary care hospitals are limited and these analyses have been important worldwide since Candida, is associated with almost $80 \%$ of all nosocomial fungal infections, representing the major cause of fungemia with high mortality rates (40\%) [11].

In this study, we examined the distribution of Candida species in cases of nosocomial Candida infections. To achieve this, we analysed the clinical records from January 2011 to May 2013 of patients who had Candida infections and were treated at Hospital Juarez de México (HJM; representative of the public health system in México).The distribution and prevalence of the relevant yeast species were determined by phenotypic methods and corroborated by PCR-RFLP of the ribosomal DNA internal transcribed spacer (rDNA-ITS) region. Susceptibility patterns to amphotericin B (AMB), fluconazole (FLU) and itraconazole (ITR) were determined using the FUNGITEST $^{\circledR}$ kit. The genetic relatedness of C. albicans isolates from hospitalized patients was determined using CAI, CAIII and CAVI multiplex PCR microsatellite assays [10].

Our study demonstrates that $C$. albicans is the prevalent Candida specie in HJM with antifungal resistance to 
fluconazole and itraconazol. Genotypic analysis revealed some genotypes present in nearly all examined C. albicans isolates, suggesting they might represent endemic strains.

\section{Materials and Methods}

\subsection{Study Design}

This was a retrospective, observational and transversal study conducted in HJM by the Medical Mycology Laboratory from January 2011 to May 2013 evaluating 167 clinical samples from patients being treated at the HJM. This hospital mainly provides primary and tertiary medical services not only to patients from Distrito Federal and Mexico State, but also to patients originating from different hospitals from the states of Hidalgo, Michoacán, Veracruz and Guerrero. The study was approved by the hospital ethics committee; however, informed consent was not requested from the patients because of the observational nature of the study. The investigators did not intervene in the standard of care, and patient's records and all information was kept confidential. The clinical data (age, gender, underlying diseases, prior use of antimicrobial and antifungal agents, malignancies, mechanical ventilation, diabetes mellitus, HIV/AIDS, invasive procedures and prematurity or low weight at birth) of patients with a clinical and laboratory diagnosis of candidiasis were collected.

Patients whose biological samples fulfilled the following inclusion criteria were considered positive for Candida infection:

- Positive Candida spp. cultures from systemic and peripheral blood subsequent to 3 days hospitalisation.

- Bronchoalveolar lavage (BAL): Respiratory tract infection is difficult to diagnose. Therefore, a combination of clinical, laboratory and radiological indicators was used to select isolates from patients who had severe respiratory diseases, exhibited symptoms such as fever, tachypnoea, dyspnoea, and chest pain, and had negative results for bacterial infection and robust positive results for yeast microscopic examination (presence of budding yeast clusters and/or filamentous morphologies).

- Urine samples, sterilely collected from patients diagnosed with a urinary tract infection (based on symptoms), with a positive microscopic exam for budding yeast clusters and/or filamentous morphologies and concomitant pyuria with counts $>100,000 \mathrm{CFU} / \mathrm{mL}$ urine. It should be noted that the fungal burden could be relevant, since a statistically significant correlation has been established between heavy candiduria $\left(>10^{4} \mathrm{CFU} / \mathrm{ml}\right.$ urine) and a high Pittet Candida colonisation index (>0.5) [12].

- Medical devices such as central venous catheter (CVC) tips and tracheal tube tips with culture counts $>20$ CFU per plate.

- Oral cavity swabs taken from creamy white, slightly raised and membranous lesions present in the mouth, gums, tonsils, soft or hard palate, and acute atrophic erythematous lesions with positive microscopic exam for budding yeast clusters and/or filamentous morphologies.

- Skin and nail scrapings and genital mucosa and pharynx swabs with positive microscopic exam for budding yeast clusters and/or filamentous morphologies as well as symptoms indicating onychomycosis, skin or mucosa lesions with erythematous borders and satellite lesions or thrush.

\subsection{Isolation and Phenotypic and Molecular Identification of Candida Isolates}

Yeast colonies were isolated from culture on Sabouraud agar (Difco, USA) or Mycosel agar (Difco, USA) incubated at $37^{\circ} \mathrm{C}$. Phenotypic species identification was based on the production of germ-tube, chlamydoconidium and hyphae morphology on cornmeal-Tween 80 agar, colony colour on CHRO Magar Candida and carbohydrate assimilation profiles generated using API 20C AUX kits (bioMérieux, France). Identification was confirmed by molecular analysis (PCR-RFLP) of the ITS1-5.8S-ITS2 rDNA fragment from all isolates. DNA extraction was performed as follows: cells grown on Sabouraud agar for $48 \mathrm{~h}$ were mechanically disrupted with glass beads in lysis buffer (2\% Triton X-100, 1\% SDS, $100 \mathrm{mM} \mathrm{NaCl,} 10 \mathrm{mM}$ Tris-HCl [pH 8.0], 1 mM EDTA [pH 8.0]), purified by chloroform/isopropanol extraction and finally dissolved in $30 \mu$ of double distilled water. Universal primers ITS1 (5'-TCC GTA GGT GAA CCT GCG G-3') and ITS4 (5'-TCC TCC GCT TAT TGA TAT GC-3') were used as previously reported [13]. The control strains were C. albicans ATCC 90028, C. tropicalis ATCC 750, C. glabrata ATCC 2001, C. parapsilosis ATCC 22019, C. krusei ATCC 6258, C. guilliermondii ATCC 6260 and C. dubliniensis CD 36. The amplified products were digested with MspI [13]; isolates identified as C. albicans were additionally digested with AvrII and then amplified with primers specific 
for C. dubliniensis: DUB-F (5'-GTATTTGTCGTTCCCCTTTC-3') and DUB-R

(5'-GTGTTGTGTGCACTAACGTC-3') [14].

\subsection{In vitro Susceptibility Test}

Susceptibility to antifungal drugs was tested using the FUNGITEST ${ }^{\circledR}$ Kit (Bio-Rad, France), a modified microtitre broth breakpoint test based on the NCCLS M27A standard. Each test plate contains six antifungal agents at two different concentrations, AMB (2 and $8 \mathrm{mg} / \mathrm{mL}$ ), FLU ( 8 and $64 \mathrm{mg} / \mathrm{ml}$ ), ITR (0.5 and $4 \mathrm{mg} / \mathrm{ml}$ ), miconazole $(0.5$ and $8 \mathrm{mg} / \mathrm{ml})$, ketoconazole $(0.5$ and $4 \mathrm{mg} / \mathrm{ml})$ and 5-fluorocytosine (5FC, 2 and $32 \mathrm{mg} / \mathrm{ml}$ ) in individual wells. Growth assessment is based on the colour change of a redox indicator. FUNGITEST results are known to correlate well with those of the NCCLS microdilution plate method; the kit has the advantage of being easy to read (coloured indicator) and use [15].

\subsection{Multiplex PCR Microsatellite Assay}

Microsatellite genotyping was performed by combining PCR buffer (20 mMTris- $\mathrm{HCl}, \mathrm{pH} \mathrm{8.4,} 50 \mathrm{mMKCl}$ ), 0.2 $\mathrm{mM}$ deoxynucleoside triphosphates, $2 \mathrm{mM} \mathrm{MgCl}_{2}, 50 \mathrm{ng}$ of genomic DNA and $0.5 \mathrm{U}$ of Taq polymerase (Applied Biosystems) in a final volume of $25 \mu$. The forward primers used in the PCR amplification were labelled with the following fluorochromes: CAI-FAM 6-carboxyfluorescein (CAA)2CTG(CAA)n), CAIII-FAM 6-carboxyfluorescein (GAA)n and CAVI-HEX hexachlorofluorescein (TAAA)n, as previously described [10]. The PCR products were run on an ABI 310 Genetic Analyser (Applied Biosystems) together with the GeneScan-500 (TAMRA) size standard (Applied Biosystems). Fragment sizes were determined automatically using the Gene Scan 3.7 Analysis software. The alleles observed for each locus were sequenced on an ABI PRISM 310 DNA automatic sequencer (Applied Biosystems). PCR product fragment sizes and allele sizes were determined automatically with Gene Scan 3.7 analysis software. Alleles were designated by the number of repeated units determined. The DP was calculated for each marker according to the Simpson index of diversity.

$$
\mathrm{DP}=1-\frac{1}{N(N-1)} \sum_{j=1}^{s} x j(x j-1)
$$

where $N$ is the number of strains, $s$ is the total number of different genotypes and $n j$ is the number of genotype $j$ strains [16]. Genetic distance between genotyped isolates was calculated using the Cavalli-Sforza and Edwards genetic distance index [17] with the Populations 1.2.32 software. A dendrogram was constructed using the unweighted pair group analysis with arithmetic mean (UPGMA) model using MEGA 5.2 software. Clonality and recombination in C. albicans isolates were verified using the Hardy-Weinberg equilibrium test and $\mathrm{F}_{\mathrm{IS}}$ value were calculated with the web version of GENEPOP (http://genepop.curtin.edu.au/) and GraphPad Prism 6.

\section{Results}

\subsection{Demographics, Clinical Characteristics and Risk Factors}

A total of 167 Candida spp. isolates were evaluated, each representing an individual infectious episode. Each case was considered to be caused by a single Candida species; three cases in which different or several yeast species were isolated were discarded from the study. Samples originated from different medical wards: internal medicine (60.5\%; cardiology, endocrinology, haematology, infectology, pneumology), intensive care unit (34.1\%; surgery, adults and paediatrics), emergency room (1.8\%) and others (3.6\%; geriatrics and dermatology). The number of isolates referred for testing each year was as follows: 65 in the first year, 62 in the second year and 40 in the 5 months of the third year. The samples were obtained from 95 male (56.9\%) and 72 (43.1\%) female patients. Patient clinical data and Candida species distribution are summarised in Table 1.

In most infection cases (141 cases, 84.4\%) the Candida isolates were identified in the BAL (40.7\%) and urine (37.1\%); only eight cases had positive blood cultures (4.8\%) and three were isolated from medical devices (1.8\%; one CVC tip and two tracheal tube tips). Isolates from superficial infections (26, 15.6\%) were identified in the oral cavity (6.0\%), pharynx swabs (6.6\%) and skin and nail scrapings (3.0\%).

Patient age ranged from 2 days to 84 years with a mean of 41 years; most cases involved patients aged 19 to 40 years $(47,28.1 \%)$ and 41 to 60 years $(60,35.9 \%)$. 
Table 1. Clinical data of patients with nosocomial candidiasis and Candida species distribution.

\begin{tabular}{|c|c|c|c|c|c|c|c|c|c|c|c|c|}
\hline Sex $(n=167)$ & & (\%) & & & & & & & & & & \\
\hline Male & 95 & (56.9) & & & & & & & & & & \\
\hline Female & 72 & $(43.1)$ & & & & & & & & & & \\
\hline \multirow[t]{2}{*}{ Age (years) } & \multicolumn{2}{|c|}{ Total } & \multicolumn{2}{|c|}{ C. albicans } & \multicolumn{2}{|c|}{ C. tropicalis } & \multicolumn{2}{|c|}{ C. parapsilosis } & \multicolumn{2}{|c|}{ C. glabrata } & \multicolumn{2}{|c|}{ Candida spp. } \\
\hline & \multicolumn{2}{|c|}{ (\%) } & \multicolumn{2}{|c|}{ (73.7) } & \multicolumn{2}{|c|}{$(15.0)$} & \multicolumn{2}{|c|}{ (5.4) } & \multicolumn{2}{|c|}{ (3.6) } & \multicolumn{2}{|c|}{ (2.4) } \\
\hline$<1$ & 5 & (3.0) & 5 & $(3.0)$ & 0 & $(0.0)$ & 0 & $(0.0)$ & 0 & $(0.0)$ & 0 & $(0.0)$ \\
\hline 1 to 18 & 25 & $(15.0)$ & 15 & $(9.0)$ & 7 & $(4.2)$ & 3 & (1.8) & 0 & $(0.0)$ & 0 & $(0.0)$ \\
\hline 19 to 40 & 47 & $(28.1)$ & 38 & (22.8) & 4 & (2.4) & 1 & $(0.6)$ & 4 & (2.4) & 0 & $(0.0)$ \\
\hline 41 to 60 & 60 & (35.9) & 43 & $(25.7)$ & 9 & (5.4) & 4 & (2.4) & 1 & $(0.6)$ & 3 & $(1.8)$ \\
\hline$>60$ & 30 & $(18.0)$ & 22 & $(13.2)$ & 5 & (3.0) & 1 & $(0.6)$ & 1 & $(0.6)$ & 1 & $(0.6)$ \\
\hline Mean age & 41 & & & & & & & & & & & \\
\hline \multicolumn{13}{|l|}{ Isolation sample } \\
\hline \multicolumn{13}{|l|}{ Deep infections } \\
\hline BAL & 68 & $(40.7)$ & 56 & (33.5) & 7 & $(4.2)$ & 2 & $(1.2)$ & 2 & $(1.2)$ & 1 & $(0.6)$ \\
\hline Urine & 62 & $(37.1)$ & 40 & $(24.0)$ & 13 & (7.8) & 4 & (2.4) & 3 & (1.8) & 2 & $(1.2)$ \\
\hline Blood & 8 & $(4.8)$ & 7 & $(4.2)$ & 0 & $(0.0)$ & 1 & $(0.6)$ & 0 & $(0.0)$ & 0 & $(0.0)$ \\
\hline Medical devices & 3 & (1.8) & 1 & $(0.6)$ & 1 & $(0.6)$ & 1 & $(0.6)$ & 0 & $(0.0)$ & 0 & $(0.0)$ \\
\hline \multicolumn{13}{|l|}{ Superficial infections } \\
\hline Swabing (genital and pharynx) & 11 & (6.6) & 6 & $(3.6)$ & 4 & $(2.4)$ & 0 & $(0.0)$ & 0 & $(0.0)$ & 1 & $(0.6)$ \\
\hline Oral cavity & 10 & $(6.0)$ & 9 & $(5.4)$ & 0 & $(0.0)$ & 0 & $(0.6)$ & 1 & $(0.6)$ & 0 & $(0.0)$ \\
\hline Skin and nail scrapping & 5 & (3.0) & 4 & $(2.4)$ & 0 & $(0.0)$ & 1 & $(0.6)$ & 0 & $(0.0)$ & 0 & $(0.0)$ \\
\hline \multicolumn{13}{|l|}{ Impatient units } \\
\hline Internal Medicine & 101 & $(60.5)$ & 71 & $(42.5)$ & 14 & (8.4) & 7 & $(4.2)$ & 5 & (3.0) & 4 & $(2.4)$ \\
\hline ICU & 57 & $(34.1)$ & 44 & (26.3) & 11 & (6.6) & 1 & $(0.6)$ & 1 & $(0.6)$ & 0 & $(0.0)$ \\
\hline Other wards & 6 & (3.6) & 5 & $(3.0)$ & 0 & $(0.0)$ & 1 & $(0.6)$ & 0 & $(0.0)$ & 0 & $(0.0)$ \\
\hline ER & 3 & (1.8) & 3 & $(1.8)$ & 0 & $(0.0)$ & 0 & $(0.0)$ & 0 & $(0.0)$ & 0 & $(0.0)$ \\
\hline \multicolumn{13}{|l|}{ Underlying diseases } \\
\hline Respiratory diseases & 55 & (32.9) & 43 & $(25.7)$ & 9 & (5.4) & 0 & $(0.0)$ & 2 & $(1.2)$ & 1 & $(0.6)$ \\
\hline Malignancies & 20 & $(12.0)$ & 13 & $(7.8)$ & 4 & (2.4) & 1 & $(0.6)$ & 1 & $(0.6)$ & 1 & $(0.6)$ \\
\hline Tuberculosis & 10 & $(6.0)$ & 8 & $(4.8)$ & 0 & $(0.0)$ & 0 & $(0.0)$ & 2 & $(1.2)$ & 0 & $(0.0)$ \\
\hline Diabetes mellitus & 7 & $(4.2)$ & 6 & (3.6) & 0 & $(0.0)$ & 1 & $(0.6)$ & 0 & $(0.0)$ & 0 & $(0.0)$ \\
\hline UTI & 7 & $(4.2)$ & 5 & $(3.0)$ & 0 & $(0.0)$ & 0 & $(0.0)$ & 1 & $(0.6)$ & 1 & $(0.6)$ \\
\hline \multicolumn{13}{|l|}{ Risk factors } \\
\hline CVC & 79 & $(47.3)$ & 61 & $(36.5)$ & 11 & (6.6) & 2 & $(1.2)$ & 3 & $(1.8)$ & 2 & $(1.2)$ \\
\hline broad-spectrum antimicrobial & 33 & $(19.8)$ & 25 & $(15.0)$ & 5 & (3.0) & 1 & $(0.6)$ & 1 & $(0.6)$ & 1 & $(0.6)$ \\
\hline Mechanical ventilation & 44 & $(26.3)$ & 35 & $(21.0)$ & 7 & $(4.2)$ & 0 & $(0.0)$ & 1 & $(0.6)$ & 1 & $(0.6)$ \\
\hline sepsis & 43 & $(25.7)$ & 36 & $(21.6)$ & 5 & (3.0) & 1 & $(0.6)$ & 0 & $(0.0)$ & 1 & $(0.6)$ \\
\hline previous use of antifungal drugs & 21 & $(12.6)$ & 16 & $(9.6)$ & 4 & $(2.4)$ & 1 & $(0.6)$ & 0 & $(0.0)$ & 0 & $(0.0)$ \\
\hline HIV/AIDS & 10 & $(6.0)$ & 7 & $(4.2)$ & 2 & $(1.2)$ & 0 & $(0.0)$ & 1 & $(0.6)$ & 0 & $(0.0)$ \\
\hline Invasive procedures & 8 & $(4.8)$ & 8 & $(4.8)$ & 1 & $(0.6)$ & 0 & $(0.0)$ & 1 & $(0.6)$ & 0 & $(0.0)$ \\
\hline autoimmunity disorders & 7 & $(4.2)$ & 5 & $(3.0)$ & 1 & $(0.6)$ & 1 & $(0.6)$ & 0 & $(0.0)$ & 0 & $(0.0)$ \\
\hline
\end{tabular}

BAL: Broncho Alveolar Lavage; ICU: Intensive Care Unit; ER: Emergency Room; UTI: Urinary Tract Infection; CVC: Central Venous Catheter. 
Underlying diseases included respiratory diseases (32.9\%; community acquired pneumonia, mechanic ventilator associated pneumonia, atypical pneumonia, obstructive chronic pulmonary disease), malignancies (12.0\%; leukaemia, lymphoma, myeloma), tuberculosis (6.0\%), type II diabetes mellitus (4.2\%) and urinary tract bacterial infections (4.2\%). The principal risk factors observed were the presence of CVC (47.3\%), use of broadspectrum antibiotics (19.8\%), mechanical ventilation (26.3\%), sepsis (25.7\%) and previous use of antifungal drugs (12.6\%).

Phenotypic and molecular analyses revealed that the most common Candida species was C. albicans (73.7\%), followed by C. tropicalis (15.0\%), C. glabrata (3.6\%) and C. parapsilosis (5.4\%) (Table 1). Other identified species (2.4\%) included the following: C. lusitaniae (two isolates), C. krusei and C. guilliermondii (one isolate each) Table 1). The phenotypic identity of all isolates (data not shown) was confirmed by comparing the resulting patterns of MspIwith the patterns of ITS-PCR RFLP for reference yeast strains after digestion with Msp I. These analyses showed that 12 of the isolates (9.3\%) had been misidentified by phenotypic methods (data not shown). Further analysis of all samples identified as C. albicans by PCR-RFLP with AvrII or by PCR using the specific primers dubF-dubRdemonstrated that $C$. dubliniensis was not present in any of the samples.

The distribution of Candida spp. did not vary significantly across the age groups, although the $41-60$ years group exhibited the highest number of non-albicans species: C. tropicalis (5.4\%), C. parapsilosis (2.4\%), C. glabrata (0.6\%) and other Candida spp. (1.8\%). Younger age groups were dominated by other species; in patients younger than 1 year, only C. albicans was isolated, while in the 1 - 18 years group, C. albicans (9.0\%) was the most abundant isolate followed by C. tropicalis (4.2\%) and C. parapsilosis (1.8\%).

\subsection{Susceptibility to Antifungal Drugs}

The in vitro susceptibility of isolates to AMB, FLU, ITR and 5FC is summarised in Table 2. The 5-fluorocytosine miconazole and ketoconazole data were omitted from further analysis since these drugs are no longer used for inpatient antifungal therapy in our hospital. Resistance tended to be more frequent among the C. albicans isolates (35\%) than the non-albicans isolates (12.5\%); however, this difference was not statistically significant.

Overall, $87.5 \%$ of the tested isolates were susceptible to AMB, $84.3 \%$ to FLU and $80 \%$ to ITR. AMB resistance was observed mainly in C. albicans (7.8\%), C. parapsilosis (3.1\%) and C. guilliermondii (1.5\%) isolates. Of the five AMB resistant $C$. albicans strains, four originated from internal medicine patients (three were isolated from BAL and one from urine) and one from the BAL of an ICU patient. Patients with BAL-isolated strains (age 18 to 54 years) exhibited severe sepsis, CVC, had received mechanical ventilation and had been hospitalised for an extended period of time; a single patient had been diagnosed with AIDS. Unexpectedly, the five AMB resistant strains were also resistant to FLU and ITR. In addition, two AMB resistant C. parapsilosis strains were identified, one from a septic patient and the other from nail scrapings. One C. guilliermondii strain isolated from nail scrapings was also AMB resistant.

Resistance to FLU was observed in C. albicans (12.5\%), C. glabrata(1.5\%) and C. krusei (1.5\%) strains; C. krusei is thought to be intrinsically resistant to FLU. As mentioned, five C. albicans strains were resistant to AMB, FLU and ITR; the remaining three strains were resistant to FLU and ITR and originated from ICU, inter-

Table 2. Resistance of Candida isolates to antifungal agents.

\begin{tabular}{|c|c|c|c|c|c|c|c|c|c|}
\hline \multirow{3}{*}{$\begin{array}{c}\text { Species } \\
\text { C. albicans }\end{array}$} & \multirow{3}{*}{$\begin{array}{c}\text { Isolates tested } \\
46\end{array}$} & \multicolumn{8}{|c|}{ Frequency of resistant isolates (\%) } \\
\hline & & \multicolumn{2}{|c|}{ Fluconazole } & \multicolumn{2}{|c|}{ Itraconazole } & \multicolumn{2}{|c|}{ Flucytosine } & \multicolumn{2}{|c|}{ Amphotericine B } \\
\hline & & 8 & $(17.4)$ & 10 & $(21.7)$ & 0 & $(0)$ & 5 & $(10.9)$ \\
\hline C. tropicalis & 7 & 0 & $(0)$ & 1 & $(14.3)$ & 0 & $(0)$ & 0 & $(0)$ \\
\hline C. parapsilosis & 3 & 0 & $(0)$ & 1 & (33.3) & 0 & $(0)$ & 2 & $(66.7)$ \\
\hline C. glabrata & 5 & 1 & $(20.0)$ & 0 & $(0)$ & 0 & (0) & 0 & $(0)$ \\
\hline C. guilliermondii & 1 & 0 & $(0)$ & 0 & (0) & 0 & $(0)$ & 1 & $(100)$ \\
\hline C. lusitaniae & 1 & 0 & $(0)$ & 1 & $(100)$ & 0 & $(0)$ & 0 & $(0)$ \\
\hline C. krusei & 1 & 1 & $(100)$ & 0 & $(0)$ & 0 & $(0)$ & 0 & (0) \\
\hline
\end{tabular}


nal medicine and emergency room patients. These strains were isolated from the blood of a male patient with disseminated tuberculosis and meningitis, the urine of a female patient with pneumonia and from the tracheal tube tip of a male patient diagnosed with pneumonia, respectively. The FLU resistant C. glabrata strain was isolated from the urine of a kidney transplant recipient who had not received previous antifungal prophylaxis with FLU. Resistance to ITR was observed in 10 C. albicans isolates (two were resistant only to ITR), and one strain of C. tropicalis and C. parapsilosis.

\subsection{Strain Differentiation of Candida albicans Isolates}

The microsatellite analysis results are summarised in Table 3. Since C. albicans is diploid, each resulting PCR fragment was defined as an allele; strains with two PCR products were considered as heterozygous, while those with a single product as homozygous. Microsatellite multiplex PCR yielded one or two PCR fragments per locus for each of the isolates. The discriminatory power (DP) of the primers was 0.95 for CAI, 0.70 for CAIII and 0.81 for CAVII. The combined DP value for the three loci is 0.98 , which is considered a reliable DP value for molecular typing studies. Genotyping analysis showed that most of the isolates were genetically related and that there was a considerable level of similarity between individual strains. Using a separate microsatellite analysis, we were able to verify the presence of 28 different alleles with the CAI primer, five alleles with CAIII and 31 with CAVI. Analysis conducted with only the CAI microsatellite (higher DP) grouped together several independent or unrelated isolates (data not shown). Moreover, when the three loci were simultaneously analysed, isolates the same CAI allele corresponded to different strains or strains with a minimal variation in CAIII or CAVI alleles.

The microsatellite analysis revealed that most of the 84 isolates share the same or similar (only one different allele) genotype (Table 3). The most common alleles in CAI were 30, 41, 35 and 28, in CAIII, 5 and 4 and CAVI 06, 09 and 03. The expected heterozygosity varied from 0.934 to 0.705 and the observed genotypes of each isolated showed significant departures from the Hardy-Weinberg equilibrium in all loci $(p<0.05)$. $\mathrm{F}_{\text {IS }}$ value for CAI and CAVI loci indicates inbreeding in the population, while negative $\mathrm{F}_{\text {IS }}$ value for CAIII loci points to out breeding (Table 4). Interestingly most of this isolates have heterozygous CAI alleles. Almost half of the isolate share the heterozygous CAIII 04:05, 05:10 and 05:08 genotypes while CAVI homozygous 06:06 and 09:09 genotypes were common. UPGMA dendrogram using Cavalli-Sforza \&Edwards genetic distance [17] shows that isolates are close related and those with the same or similar genotype cluster together (Figure 1).

\section{Discussion}

To the best of our knowledge, this is the first surveillance study of Candida species obtained from patients hospitalised in Hospital Juarez de México (HJM). In this study we sought to determine the relative prevalence of $C$. albicans and non-albicans Candida spp. in different patient specimens. All isolates were identified using a highly sensitive and specific molecular method; this precise identification ensured the reliability of our distribution data.

The biological specimens from which Candida spp. were most commonly isolated were BAL (40.7\%) and urine (37.1\%) samples originating from anatomical locations that are colonised by yeast. Only samples with a positive microscopic examination and clinical evidence of infection were included in this study as opposed to other studies that have used culturing techniques for verification, while discarding infection due to incapacity to determine if its colonization or infection growth. Most of the virulence factors of Candida species are expressed in filamentous morphologies [18], therefore the microscopic examination could help in discrimination and diagnosis. Further studies may determine the relationship of expression of virulence factor genes in Candida yeast and filamentous morphologies biological samples (in vivo).

This study shows that C. albicans was the most prevalent isolate (73.7\%), in accordance with previous reports from various countries [19]. However, the abundance of this species does not agree with other global studies demonstrating a significant increase in the prevalence of non-albicans species over the past three decades. Other abundant species identified in this study were C. tropicalis (15\%) followed by C. parapsilosis (5.4\%). There was a clear predominance of $C$. albicans in children while non-albicans species were present mainly in adults aged 19 - 60 years. No significant differences were found between male and female patients.

Two previous studies conducted in Mexico describe species distribution and antifungal susceptibilities among Candida isolates obtained from blood cultures collected in different tertiary hospitals: two hospitals in the south 
Table 3. Microsatellite typing of C. albicans isolates from Hospital Juarez de Mexico. Isolates with the same genotype or with a single allele difference are in bold with grey background.

\begin{tabular}{|c|c|c|c|c|c|c|}
\hline \multirow[b]{2}{*}{ Isolate } & \multicolumn{3}{|c|}{ Microsatellite alleles } & \multicolumn{3}{|c|}{ Sample's information } \\
\hline & CAI & CAIII & CAIV & Ward & Diagnosis & Sample \\
\hline $150-13$ & $48: 48$ & $05: 10$ & 09:09 & $\mathrm{IM}$ & Diabetes mellitus II & Urine \\
\hline 223-11 & $37: 52$ & 05:10 & 09:20 & IM & HIV+ & BAL \\
\hline 223-12 & $37: 52$ & 05:10 & 09:20 & IM & CAP & Urine \\
\hline 094-13 & $37: 46$ & $05: 10$ & 08:08 & $\mathrm{IM}$ & ALL & Swab \\
\hline $462-12$ & $36: 36$ & 05:10 & $07: 17$ & $\mathrm{IM}$ & Diabetes mellitus II & Urine \\
\hline 391-11 & $35: 47$ & 05:10 & 09:09 & IM & Cystic fibrosis & BAL \\
\hline $393-11$ & $35: 47$ & 05:10 & 09:09 & IM & HIV+ & Blood \\
\hline 069-12 & $35: 47$ & $10: 10$ & 09:09 & IM & Lymphoid Leukemia & Urine \\
\hline $045-13$ & $35: 43$ & $10: 10$ & 09:14 & ICU & Septic shock & Urine \\
\hline $430-12$ & $35: 41$ & 05:08 & 06:06 & Others & Onychomycosis & Skin scrapping \\
\hline $469-12$ & $35: 41$ & 05:08 & 06:06 & ICU & Septic shock & Urine \\
\hline $963-10$ & $35: 40$ & 04:04 & 09:09 & $\mathrm{IM}$ & Tb, meningitis & Blood \\
\hline $483-10$ & $35: 38$ & $10: 10$ & 19:19 & $\mathrm{IM}$ & Sepsis & BAL \\
\hline 043-11 & $35: 35$ & 05:10 & 09:09 & Ped. ICU & Fungal sepsis & Urine \\
\hline $047-13$ & $35: 35$ & $10: 10$ & 09:09 & IM & $\mathbf{T b}$ & BAL \\
\hline $167-12$ & $35: 35$ & 05:10 & 09:09 & Ped. ICU & HIV+, Balanitis, Onychomycosis & Skin scrapping \\
\hline 478-12 & $34: 52$ & 05:10 & 09:09 & IM & Diabetes mellitus II & Urine \\
\hline 493-11 & $33: 41$ & 05:08 & 06:06 & IM & SLE, VAP & BAL \\
\hline $398-12$ & $34: 41$ & 05:08 & 06:06 & ICU & Septic shock & BAL \\
\hline 483-09 & 34:39 & 05:10 & 09:12 & IM & Sepsis & BAL \\
\hline $492-09$ & $33: 41$ & 08:08 & 06:06 & IM & Atypical CAP & BAL \\
\hline 922-10 & $33: 41$ & 04:04 & 06:06 & Others & septic shock & Urine \\
\hline 059-12 & $33: 41$ & 04:05 & 05:06 & IM & CAP & BAL \\
\hline $141-12$ & $33: 41$ & 04:05 & 06:06 & ICU & Pneumonia & BAL \\
\hline $394-12$ & $33: 41$ & 05:08 & 06:06 & ICU & SLE & BAL \\
\hline $457-12$ & $33: 41$ & 04:05 & 06:06 & ICU & ARDS & Urine \\
\hline 216-13 & $33: 41$ & 04:05 & 06:06 & IM & Septic shock & Urine \\
\hline 219-13 & $33: 41$ & 04:05 & 06:06 & IM & Sepsis & Urine \\
\hline $120-11$ & $33: 40$ & 04:05 & 09:09 & IM & ALL Pre B & BAL \\
\hline 225-12S & 33:33 & 04:05 & 05:06 & IM & Bacterial endocarditis & Swab \\
\hline $225-12 U$ & $33: 33$ & 04:05 & 06:06 & IM & Bacterial endocarditis & Urine \\
\hline 027-11 & $32: 42$ & 04:05 & 06:06 & ICU & $\mathbf{T b}$ & BAL \\
\hline 119-11 & $32: 41$ & 05:08 & 06:06 & ICU & Pneumonia & BAL \\
\hline 016-12 & $32: 41$ & 05:08 & 06:06 & IM & Sepsis & BAL \\
\hline $161-12$ & $32: 41$ & 05:08 & 06:06 & IM & Pneumonia, SLE & BAL \\
\hline $162-12$ & $32: 41$ & 05:10 & 06:06 & IM & Pneumonia & Urine \\
\hline $164-12$ & $32: 41$ & 05:08 & 06:06 & IM & Ascites & Urine \\
\hline $166-12$ & $32: 42$ & 04:05 & 06:06 & ICU & Lung sepsis & BAL \\
\hline $334-12$ & $32: 42$ & 04:05 & 06:06 & IM & $\mathbf{T b}$ & BAL \\
\hline 396-12 & $32: 41$ & 05:08 & 06:06 & IM & $\mathbf{T b}$ & BAL \\
\hline 439-12 & $32: 42$ & 04:05 & 06:06 & ICU & Fungal sepsis & BAL \\
\hline
\end{tabular}




\begin{tabular}{|c|c|c|c|c|c|c|}
\hline \multicolumn{7}{|l|}{ Continued } \\
\hline $111-12$ & $31: 39$ & $05: 10$ & $20: 20$ & ICU & Lung sepsis & BAL \\
\hline 103-12 & $30: 40$ & 04:05 & 06:06 & IM & Sepsis & BAL \\
\hline $498-11$ & $30: 30$ & 04:05 & $27: 27$ & $\mathrm{IM}$ & Oropharyngeal candidiasis & Swab \\
\hline $220-13$ & $30: 30$ & 05:05 & 03:06 & $\mathrm{IM}$ & Pneumonia & Urine \\
\hline $161-13$ & $30: 30$ & 04:05 & 03:30 & $\mathrm{IM}$ & Pneumonia & BAL \\
\hline $364-12$ & $29: 55$ & $05: 10$ & 09:09 & ICU & Atypical pneumonia, ARDS & Blood \\
\hline 498-09 & 28:33 & 08:08 & 06:06 & IM & Myeloma, oral candidosis & Swab \\
\hline 217-11 & 28:33 & 07:08 & 07:07 & IM & $\mathbf{T b}$ & BAL \\
\hline $360-12$ & 28:33 & 07:08 & 07:10 & IM & Pneumonia, scleroderma & BAL \\
\hline $366-12$ & 28:33 & 07:08 & 07:07 & IM & Sepsis & Urine \\
\hline $108-13$ & 28:33 & 07:08 & 07:10 & ICU & Fungemia & Blood \\
\hline $198-12$ & $28: 28$ & 05:05 & 09:15 & ICU & CAP & BAL \\
\hline $175-13$ & $28: 28$ & 07:08 & 07:07 & ICU & Sepsis & Urine \\
\hline $401-12$ & $27: 27$ & $05: 10$ & 09:09 & IM & CAP, HIV+ & BAL \\
\hline $390-12$ & $26: 30$ & $05: 10$ & 07:07 & $\mathrm{IM}$ & $\mathrm{Tb}$ & BAL \\
\hline $485-12$ & $25: 28$ & 04:04 & $06: 16$ & ICU & Sepsis & BAL \\
\hline 501-09 & $24: 30$ & 04:05 & $37: 37$ & IM & Wagner's granulomatosis & Swab \\
\hline $451-09$ & $24: 30$ & 05:05 & 03:17 & $\mathrm{IM}$ & Oral candidiasis, pneumonia & Swab \\
\hline 274-11 & $24: 30$ & 04:05 & 03:38 & Ped. ICU & Sepsis, severe neutropenia & Urine \\
\hline 318-12 & $24: 30$ & 04:05 & 33:33 & ICU & CAP & BAL \\
\hline $423-12$ & $24: 30$ & 04:05 & 03:37 & IM & CAP & BAL \\
\hline 174-13 & $24: 30$ & 04:05 & 03:26 & ICU & Septic shock & Urine \\
\hline $975-10$ & $24: 29$ & 04:05 & $27: 44$ & $\mathrm{IM}$ & ALL & Swab \\
\hline $355-12$ & $24: 29$ & 04:05 & $37: 39$ & $\mathrm{IM}$ & $\mathrm{Tb}$ & BAL \\
\hline $092-11$ & $24: 28$ & 04:04 & 06:06 & ICU & Sepsis & BAL \\
\hline $527-11$ & $23: 30$ & 04:05 & 03:03 & IM & $\mathbf{T b}$ & BAL \\
\hline $105-12$ & $23: 30$ & 04:05 & 03:03 & ICU & Leukemia & BAL \\
\hline $375-12$ & $23: 30$ & 04:05 & 03:35 & ICU & ARDS & Urine \\
\hline 443-12 & $23: 30$ & 04:05 & 03:36 & IM & Nosocomial pneumonia & BAL \\
\hline $503-12$ & $23: 30$ & 04:05 & 03:34 & ICU & Septic shock & BAL \\
\hline 203-13 & $30: 30$ & 04:05 & 03:36 & ICU & Septic shock, ARDS & Urine \\
\hline 092-13 & $23: 30$ & 04:05 & $47: 58$ & ICU & Pneumonia, ARDS & BAL \\
\hline $482-09$ & $23: 23$ & 04:05 & $42: 46$ & IM & $\mathrm{Tb}$ & BAL \\
\hline $402-12 \mathrm{H}$ & 19:26 & 04:05 & 06:06 & Ped. ICU & Pneumonia, ARDS, gastroschisis & Blood \\
\hline $402-12 U$ & $18: 26$ & 04:05 & 06:06 & Ped. ICU & Pneumonia, ARDS, gastroschisis & Urine \\
\hline 854-10 & $18: 27$ & 04:05 & 06:06 & IM & Bacterial meningitis & Urine \\
\hline $387-12$ & $18: 28$ & 04:05 & 06:06 & ICU & Acute abdomen & BAL \\
\hline 403-12 & $18: 27$ & 04:05 & 06:06 & ICU & Sepsis & BAL \\
\hline $410-12$ & $18: 26$ & 05:10 & 06:06 & Ped. ICU & Pneumonia, candidemia & Blood \\
\hline 451-12 & $18: 27$ & 04:05 & 06:06 & ICU & Sepsis & BAL \\
\hline $463-12$ & $18: 26$ & 04:05 & 06:06 & EXT & Diabetes mellitus II, skin candidosis & Skin scrapping \\
\hline 474-12 & $18: 28$ & 04:05 & 06:06 & IM & Sepsis, ARDS & BAL \\
\hline 083-13 & 18:18 & 04:05 & 06:06 & IM & CAP & Urine \\
\hline
\end{tabular}

${ }^{*}$ Number 1 and 2 next to the initials of each antifungal represent the lower and high concentration of the drug in the well of the FUNGITEST ${ }^{\circledR}$ plate. ${ }^{* * *} 0$ represents that isolate didn't grow after 72 hours, 2 represents grow before 72 hours of incubation. -for isolates with missing antifungal sensitivity data. HIV: Human Immunodeficiency Virus; CAP: Community Acquired Pneumonia; Tb: Tuberculosis; ALL; Acute Lymphocytic Leukemia; SLE: Systemic Lupus Erythematosus; VAN: Ventilator Associated Pneumonia; ICU: Intensive Care Unit; Ped. ICU: Pediatric ICU; ARDS: Acute Respiratory Distress Syndrome; BAL: Broncho-Alveolar Lavage. 


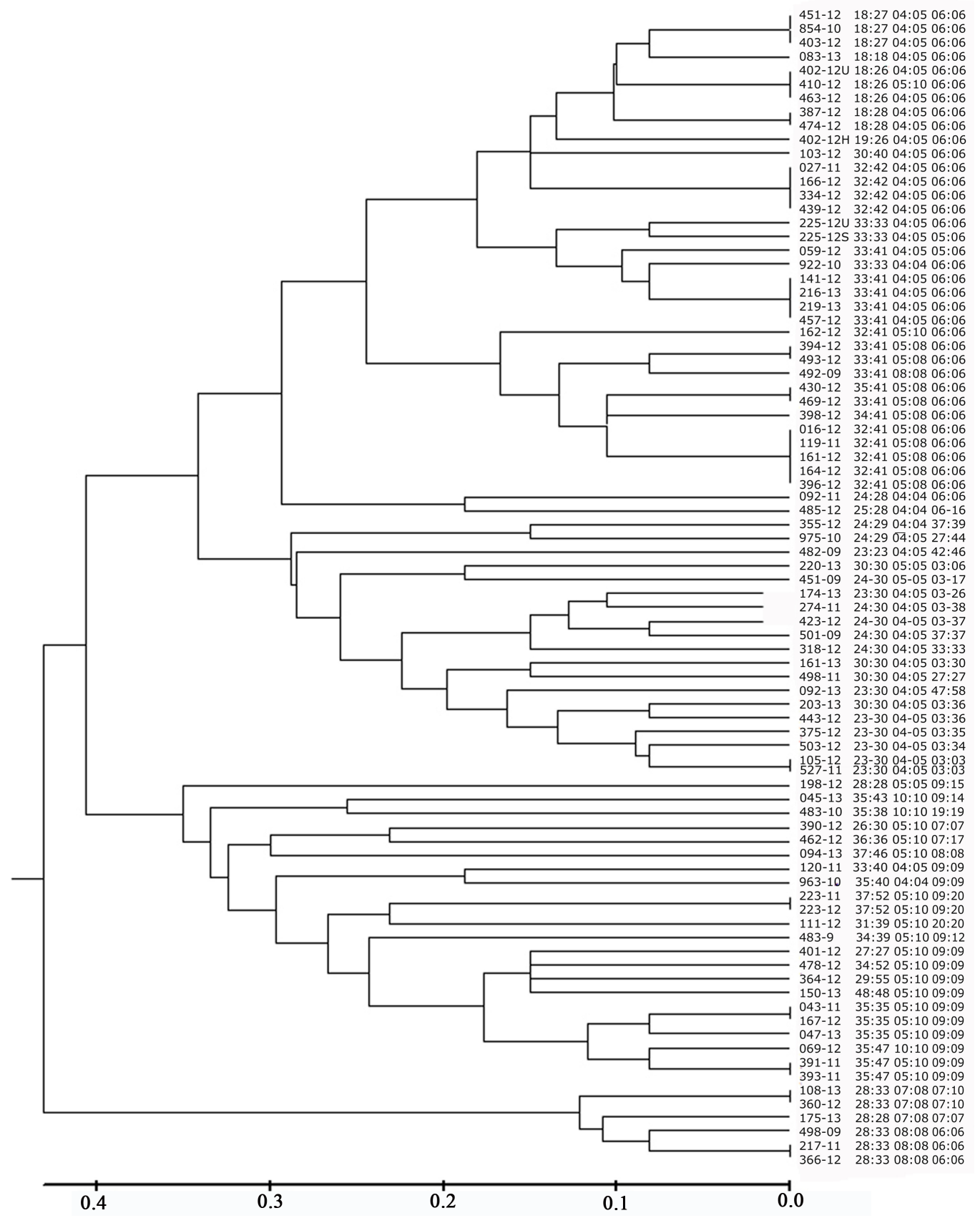

Figure 1. Dendrogram inferred using the UPGMA method in MEGA 5.2 software. The tree is drawn to scale, with branch lengths in the same units as those of the Cavalli, Sforza \& Edwards (1987) genetic distance calculated by Populations 1.2.23. Scale represents genetic similitude between isolates. As can be seen, nodes of isolates with similar o some genotypes branch at values above 0.1 of genetic similitude scales.

Table 4. Genetic diversity (heterozygosity) for each locus and $\mathrm{F}_{\mathrm{IS}}$ value in C. albicans isolates.

\begin{tabular}{|c|c|c|c|c|c|c|}
\hline \multirow{2}{*}{ Locus } & \multicolumn{2}{|c|}{ CAI } & \multicolumn{2}{|c|}{ CAIII } & \multicolumn{2}{|c|}{ CAIV } \\
\hline & $\mathrm{He}$ & Ho & $\mathrm{He}$ & Но & $\mathrm{He}$ & Ho \\
\hline & 0.934 & 0.809 & 0.705 & 0.845 & 0.775 & 0.298 \\
\hline $\mathrm{F}_{\mathrm{IS}}$ & 0.1340 & & -0.2007 & & 0.6175 & \\
\hline
\end{tabular}


of Mexico City (SMC) [20], and five tertiary-level teaching hospitals in Monterrey state (MS) [21]. These studies report frequency of Candida species from bloodstream infections; however they differ importantly in the distribution of Candida species. In Monterrey, C. parapsilopsis (37.9\%) was observed as the prevalent specie followed by C. albicans (31.9\%) while in SMC was C. albicans (46\%) followed by C. tropicalis (26\%).

It is important to emphasise that $C$. tropicalis infections are more common in patients with severe neutropenia and haematological cancer [22], this may explain the higher number of $C$. tropicalis isolates in the SMC study, which included samples from the National Cancer Institute, compared with our study, which contained a low number of samples from patients with haematological cancers.

Interestingly, there is significant geographic variation in the prevalence of non-albicans Candida NAC specie [23] [24] C. glabrata and C. parapsilosis are more frequent in North America compared with Latin America, while C. tropicalis is an emerging pathogen in hospitals in Latin America, certain Asian countries (Singapore and Hong Kong) [25] [26] and is the first non-albicans species identified in Pakistan [27].

One important finding of our study is that $C$. dubliniensis was not identified by molecular analysis. C. albicans and C. dubliniensis are closely related species that can be misidentified using phenotypic methods; however, both the PCR results using specific primers for C. dubliniensis (DUB) and the RFLP analysis with different enzymes clearly demonstrated the absence of $C$. dubliniensis in our C. albicans samples. This finding is surprising since a number of studies have reported infections due to $C$. dubliniensis in different geographic regions (Europe, North America, Argentina, Australia and Singapore) [28] [29].

Antifungal susceptibility analysis confirmed that resistance to AMB, FLU and ITR is an important characteristic of the HJM C. albicans isolates. Many studies have reported that resistant C. albicans and C. parapsilosis strains are rarely isolated [30] [31]; however, the rise in resistance against these important antifungal drugs has been increasingly associated with the use of these antifungal agents, resulting in the selection of specific resistant isolates. The widespread use of FLU as a prophylactic treatment and the preference for using AMB because of its relatively low cost, may promote the increase in the number of resistant isolates among these pathogens. Previous studies have demonstrated that resistance to AMB is not common in Candida spp., except for C. lusitaniae [32]; however, this specie was poorly represented in the current study. Our data indicate that 8 of 10 ITR resistant C. albicans strains were also resistant to FLU. A similar pattern has been observed in other studies, and it is known that resistance is mediated via specific ATP binding cassette (ABC) family transporters that afford cross resistance to azole antifungal drugs.

Microsatellites, or simple sequence repeats (SSRs), are tandem 1 - 6 bp nucleotide repeats dispersed throughout the genome and can be utilised for strain typing and epidemiological studies. Several polymorphic microsatellite loci have been identified for C. albicans including the C. albicans specific CAI, CAIII and CAVI. These loci are located in noncoding regions, and thus are under low selective pressures resulting in a high degree of polymorphism. These markers are stable and show high levels of robustness and specificity for C. albicans inter-strain differentiation; they have been used as a typing system for characterising clinical isolates and identifying nosocomial transmission of C. albicans [10] [33].

Our analysis with CAI, CAIII and CAVI of independent isolates (Table 3) revealed a high level of polymorphism; 56 isolates had identical CAI, CAIII and CAVI genotypes or had minor changes in the CAI or CAVI loci. Each of the isolates originated from a single patient, except for four isolates, 225-15S, 225-12U (two samples of the same patient) and , 402-12H and 402-12U (two samples of the same ICU patient), and most of these patients were hospitalised in the ICU and IM wards. It is important to emphasise that these same-genotype isolates were recovered from different samples and therefore different patients. Analysis of the 225-12 and 402-12 isolate pairs showed that they have almost identical genotypes, confirming that only one strain was present in each of the patients during Candida infection. Moreover, the presence of isolates with minimal variation in all microsatellite loci suggests the occurrence of an adaptation to environmental conditions as part of a microevolution process, as previously described [34].

Population analysis suggests that the genotyped C. albicans isolates represent a clonal population (according to the Hardy-Weinberg test). The $\mathrm{F}_{\mathrm{IS}}$ values further confirm the CAI and CAVI loci results that indicate "inbreeding" and clonal expansion of conserved genotypes by mitotic reproduction (C. albicans lacks a conventional sexual cycle) [35]. However, the $\mathrm{F}_{\mathrm{IS}}$ value for the CAIII locus seems somewhat inconsistent, and points to the prevalence of non-clonal C. albicans isolates. It is important to note, however, that the CAIII primer has a lower DP value, and therefore this marker may be not suitable for genotypic and genetic analysis. Moreover, the CAIII primer amplifies a microsatellite sequence in chromosome 5 , and several studies have demonstrated that 
loss of heterozygosity is common in this chromosome [16], resulting in homozygous genotypes for chromosome 5 genes (including the CAIII microsatellite). For these reasons, the CAIII $\mathrm{F}_{I S}$ value was subsequently discarded.

Exogenous nosocomial transmission of Candida spp. has been reported [36], however endogenous colonization is responsible for severe candidiasis [37]. The data reported in this study may represent endemic Candida strains in the hospital; however, the clinical data could not demonstrate that patients shared possible Candida transmission sources, including physical space, healthcare workers, caretakers or food. To address these variables, a prospective study should be conducted that would recover isolates from the same ward and time period and examine the prevalence of identical genotype isolates. It is possible that the $C$. albicans isolates identified in our current study may represent endemic isolates and not nosocomial strains; however, since this is the first study of its kind conducted in Mexico, further studies will be required to obtain additional epidemic data and to prevent for fungal drug resistance in the hospital environment.

\section{Conclusions}

C. albicans was the most prevalent species identified in patients hospitalised in the HJM, which was representative of conditions in the public health care system, in contrast with the decreasing worldwide prevalence of this pathogen. The distribution of Candida spp. isolates has been found to vary in different geographical areas, most likely due to the considerable effect of underlying conditions and prophylactic antifungal therapy agents on local epidemiology. Horizontal transmission may also affect species distribution.

The microsatellite multiplex PCR-based system enables high volume typing of isolates, and is therefore useful in epidemiological studies and in the identification of nosocomial and endemic C. albicans isolates. Genotypic analysis revealed the presence of a number of genotypes in nearly all examined C. albicans isolates, suggesting they might represent endemic strains. The current study is limited in that it is performed at a single México City hospital, and thus the results cannot be generalised. This study is very important in our hospital because further analysis of the Candida spp. will provide the changes in distribution of the different species due to prophylactic and antifungic treatments.

\section{Acknowledgements}

This work was supported by grant PAPIIT-IN214013 from Universidad NacionalAutónoma de México. The statements herein are solely the responsibility of the authors. We thank technical assistance of Angelica L. Serrano-Ahumada.

\section{Conflict of Interests}

The authors declare that no conflict of interest exists regarding the publication of this paper.

\section{References}

[1] Chang, A., Neofytos, D. and Horn, D. (2008) Candidemia in the 21st Century. Future Microbiology, 3, 463-472. http://dx.doi.org/10.2217/17460913.3.4.463

[2] Vincent, J.L., Rello, J., Marshall, J., Silva, E., Anzueto, A., Martin, C.D., Moreno, R., Lipman, J., Gomersall, C., Sakr, Y. and Reinhart, K., E. I. G. O. Investigators (2009) International Study of the Prevalence and Outcomes of Infection In Intensive Care Units. Journal of the American Medical Association, 302, 2323-2329. http://dx.doi.org/10.1001/jama.2009.1754

[3] Cornely, O.A., Bassetti, M., Calandra, T., Garbino, J., Kullberg, B.J., Lortholary, O., Meersseman, W., Akova, M., Arendrup, M.C., Arikan-Akdagli, S., Bille, J., Castagnola, E., Cuenca-Estrella, M., Donnelly, J.P., Groll, A.H., Herbrecht, R., Hope, W.W., Jensen, H.E., Lass-Florl, C., Petrikkos, G., Richardson, M.D., Roilides, E., Verweij, P.E., Viscoli, C. and Ullmann, A.J., E. F. I. S. Group (2012) ESCMID* Guideline for the Diagnosis and Management of Candida Diseases 2012: Non-Neutropenic Adult Patients. Clinical Microbiology and Infection, 18, 19-37. http://dx.doi.org/10.1111/1469-0691.12039

[4] Chen, Y.L., Yu, S.J., Huang, H.Y., Chang, Y.L., Lehman, V.N., Silao, F.G., Bigol, U.G., Bungay, A.A., Averette, A. and Heitman, J. (2014) Calcineurin Controls Hyphal Growth, Virulence, and Drug Tolerance of Candida Tropicalis. Eukaryotic Cell, 13, 844-854. http://dx.doi.org/10.1128/EC.00302-13

[5] Naglik, J.R., Moyes, D.L., Wachtler, B. and Hube, B. (2011) Candida Albicans Interactions with Epithelial Cells and Mucosal Immunity. Microbes and Infection, 13, 963-976. http://dx.doi.org/10.1016/j.micinf.2011.06.009 
[6] Czaika, V., Nenoff, P., Glockner, A., Fegeler, W., Becker, K. and Schmalreck, A.F. (2013) Epidemiology and Changes in Patient-Related Factors from 1997 to 2009 in Clinical Yeast Isolates Related to Dermatology, Gynaecology, and Paediatrics. International Journal of Microbiology, 2013, Article ID: 703905. http://dx.doi.org/10.1155/2013/703905

[7] Mousavi, S.A.A., Khalesi, E., Bonjar, G.H.S., Aghighi, S., Sharifi, F. and Aram, F. (2007) Rapid Molecular Diagnosis For-Candida Species Using PCR-RFLP. Biotechnology, 6, 583-587. http://dx.doi.org/10.3923/biotech.2007.583.587

[8] Abdulrahim, M.H., McManus, B.A., Flint, S.R. and Coleman, D.C. (2013) Genotyping Candida albicans from Candida Leukoplakia and Non-Candida Leukoplakia Shows No Enrichment of Multilocus Sequence Typing Clades but Enrichment of ABC Genotype C in Candida Leukoplakia. PLOS ONE, 8, e73738. http://dx.doi.org/10.1371/journal.pone.0073738

[9] Lyon, J.P., Moraes, K.C., Moreira, L.M., Aimbire, F. and de Resende, M.A. (2010) Candida albicans: Genotyping Methods and Clade Related Phenotypic Characteristics. Brazilian Journal of Microbiology, 41, 841-849.

[10] Sampaio, P., Gusmao, L., Correia, A., Alves, C., Rodrigues, A.G., Pina-Vaz, C., Amorim, A. and Pais, C. (2005) New Microsatellite Multiplex PCR for Candida albicans Strain Typing Reveals Microevolutionary Changes. Journal of Clinical Microbiology, 43, 3869-3876. http://dx.doi.org/10.1128/JCM.43.8.3869-3876.2005

[11] Mikulska, M., Del Bono, V., Ratto, S. and Viscoli, C. (2012) Occurrence, Presentation and Treatment of Candidemia. Expert Review of Clinical Immunology, 8, 755-765. http://dx.doi.org/10.1586/eci.12.52

[12] Chabasse, D. (2001) Intérêt de la numération des levures dans les urines. Revue de la littérature et résultats préliminaires d'une enquête multicentrique réalisée dans 15 centres hospitaliers universitaires. Annales Françaises d'Anesthésie et de Réanimation, 20, 400-406. http://dx.doi.org/10.1016/S0750-7658(01)00376-8

[13] Mirhendi, H., Makimura, K., Khoramizadeh, M. and Yamaguchi, H. (2006) A One-Enzyme PCR-RFLP Assay for Identification of Six Medically Important Candida Species. Nihon Ishinkin Gakkai Zasshi, 47, 225-229. http://dx.doi.org/10.3314/jimm.47.225

[14] Donnelly, S. M., Sullivan, D. J., Shanley, D. B. and Coleman, D.C. (1999) Phylogenetic Analysis and Rapid Identification of Candida dubliniensis Based on Analysis of ACT1 Intron and Exon Sequences. Microbiology, 145, 1871-1882. http://dx.doi.org/10.1099/13500872-145-8-1871

[15] Witthuhn, F., Toubas, D., Beguinot, I., Aubert, D., Rouger, C., Remy, G. and Pinon, J.M. (1999) Evaluation of the Fungitest Kit by Using Strains from Human Immunodeficiency Virus-Infected Patients: Study of azole Drug Susceptibility. Journal of Clinical Microbiology, 37, 864-866.

[16] Hunter, P.R. and Gaston, M.A. (1988) Numerical Index of the Discriminatory Ability of Typing Systems: An Application of Simpson's Index of Diversity. Journal of Clinical Microbiology, 26, 2465-2466.

[17] Cavalli-Sforza, L.L. and Edwards, A.W. (1967) Phylogenetic Analysis. Models and Estimation Procedures. The American Journal of Human Genetics, 19, 233-257.

[18] Kumamoto, C.A. and Vinces, M.D. (2005) Contributions of Hyphae and Hypha-co-Regulated Genes to Candida albicans Virulence. Cellular Microbiology, 7, 1546-1554. http://dx.doi.org/10.1111/j.1462-5822.2005.00616.X

[19] Nucci, M., Queiroz-Telles, F., Tobon, A.M., Restrepo, A. and Colombo, A.L. (2010) Epidemiology of Opportunistic Fungal Infections in Latin America. Clinical Infectious Diseases, 51, 561-570. http://dx.doi.org/10.1086/655683

[20] Corzo-Leon, D.E., Alvarado-Matute, T., Colombo, A.L., Cornejo-Juarez, P., Cortes, J., Echevarria, J.I., Guzman-Blanco, M., Macias, A.E., Nucci, M., Ostrosky-Zeichner, L., Ponce-de-Leon, A., Queiroz-Telles, F., Santolaya, M.E., Thompson-Moya, L., Tiraboschi, I.N., Zurita, J. and Sifuentes-Osornio, J. (2014) Surveillance of Candida spp Bloodstream Infections: Epidemiological Trends and Risk Factors of Death in Two Mexican Tertiary Care Hospitals. PLoS ONE, 9, e97325. http://dx.doi.org/10.1371/journal.pone.0097325

[21] Gonzalez, G.M., Elizondo, M. and Ayala, J. (2008) Trends in Species Distribution and Susceptibility of Bloodstream Isolates of Candida Collected in Monterrey, Mexico, to Seven Antifungal Agents: Results of a 3-Year (2004 to 2007) Surveillance Study. Journal of Clinical Microbiology, 46, 2902-2905. http://dx.doi.org/10.1128/JCM.00937-08

[22] Chen, C.Y., Huang, S.Y., Tsay, W., Yao, M., Tang, J.L., Ko, B.S., Chou, W.C., Tien, H.F. and Hsueh, P.R. (2012) Clinical Characteristics of Candidaemia in Adults with Haematological Malignancy, and Antimicrobial Susceptibilities of the Isolates at a Medical Centre in Taiwan, 2001-2010. International Journal of Antimicrobial Agents, 40, 533-538. http://dx.doi.org/10.1016/j.ijantimicag.2012.07.022

[23] Yamamoto, A.C.A., de Paula, C.R., Dias, L.B., Tadano, T., Martins, E.R., Amadio, J.V. and Hahn, R.C. (2012) Epidemiological and Clinical Characteristics of Nosocomial Candidiasis in University Hospitals in Cuiaba-Mato Grosso, Brazil. Revista Iberoamericana de Micologíal, 29, 164-168. http://dx.doi.org/10.1016/j.riam.2012.01.001

[24] Quindos, G. (2014) Epidemiology of Candidaemia and Invasive Candidiasis. A Changing Face. Revista Iberoamericana de Micología, 31, 42-48. http://dx.doi.org/10.1016/j.riam.2013.10.001

[25] Chai, Y.A., Wang, Y., Khoo, A.L., Chan, F.Y., Chow, C., Kumarasinghe, G., Singh, K. and Tambyah, P.A. (2007) Predominance of Candida tropicalis Bloodstream Infections in a Singapore Teaching Hospital. Medical Mycology, 45, 
435-439. http://dx.doi.org/10.1080/13693780701385868

[26] Yap, H.Y., Kwok, K.M., Gomersall, C.D., Fung, S.C., Lam, T.C., Leung, P.N., Hui, M. and Joynt, G.M. (2009) Epidemiology and Outcome of Candida Bloodstream Infection in an Intensive Care Unit in Hong Kong. Hong Kong Medical Journal, 15, 255-261.

[27] Farooqi, J.Q., Jabeen, K., Saeed, N., Iqbal, N., Malik, B., Lockhart, S.R., Zafar, A., Brandt, M.E. and Hasan, R. (2013) Invasive Candidiasis in Pakistan: Clinical Characteristics, Species Distribution and Antifungal Susceptibility. Journal of Medical Microbiology, 62, 259-268.

[28] Costa, A.R., Silva, F., Henriques, M., Azeredo, J., Oliveira, R. and Faustino, A. (2010) Candida Clinical Species Identification: Molecular and Biochemical Methods. Annals of Microbiology, 60, 105-112. http://dx.doi.org/10.1007/s13213-009-0007-6

[29] Khan, Z., Ahmad, S., Joseph, L. and Chandy, R. (2012) Candida dubliniensis: An Appraisal of Its Clinical Significance as a Bloodstream Pathogen. PLoS ONE, 7, e32952. http://dx.doi.org/10.1371/journal.pone.0032952

[30] Garcia-Agudo, L., Garcia-Martos, P., Marin-Casanova, P. and Rodriguez-Iglesias, M. (2012) [Susceptibility to Fluconazole of Clinical Interest Yeasts: New Breakpoints]. Revista Española de Quimioterapia, 25, 266-268.

[31] Pfaller, M.A. and Diekema, D.J. (2012) Progress in Antifungal Susceptibility Testing of Candida spp. by Use of Clinical and Laboratory Standards Institute Broth Microdilution Methods, 2010 to 2012. Journal of Clinical Microbiology, 50, 2846-2856. http://dx.doi.org/10.1128/JCM.00937-12

[32] Peyron, F., Favel, A., Michel-Nguyen, A., Gilly, M., Regli, P. and Bolmstrom, A. (2001) Improved Detection of Amphotericin B-Resistant Isolates of Candida lusitaniae by Etest. Journal of Clinical Microbiology, 39, 339-342. http://dx.doi.org/10.1128/JCM.39.1.339-342.2001

[33] Spampinato, C. and Leonardi, D. (2013) Molecular Fingerprints to Identify Candida Species. BioMed Research International, 2013, Article ID: 923742. http://dx.doi.org/10.1155/2013/923742

[34] Amouri, I., Sellami, H., Abbes, S., Hadrich, I., Mahfoudh, N., Makni, H. and Ayadi, A. (2012) Microsatellite Analysis of Candida Isolates from Recurrent Vulvovaginal Candidiasis. Journal of Medical Microbiology, 61, 1091-1096.

[35] Rustchenko, E. (2007) Chromosome Instability in Candida albicans. FEMS Yeast Research, 7, 2-11. http://dx.doi.org/10.1111/j.1567-1364.2006.00150.x

[36] Pfaller, M.A., Messer, S.A., Houston, A., Rangel-Frausto, M.S., Wiblin, T., Blumberg, H.M., Edwards, J.E., Jarvis, W., Martin, M.A., Neu, H.C., Saiman, L., Patterson, J.E., Dibb, J.C., Roldan, C.M., Rinaldi, M.G. and Wenzel, R.P. (1998) National Epidemiology of Mycoses Survey: A Multicenter Study of Strain Variation and Antifungal Susceptibility among Isolates of Candida Species. Diagnostic Microbiology and Infectious Disease, 31, 289-296. http://dx.doi.org/10.1016/S0732-8893(97)00245-9

[37] Voss, A., Hollis, R.J., Pfaller, M.A., Wenzel, R.P. and Doebbeling, B.N. (1994) Investigation of the Sequence of Colonization and Candidemia in Nonneutropenic Patients. Journal of Clinical Microbiology, 32, 975-980. 